\title{
The safety and efficacy of EGF-based cream for the prevention of radiotherapy-induced skin injury: results from a multicenter observational study
}

\author{
Hyun-Cheol Kang, MD, ${ }^{1,2}$ Seung-Do Ahn, MD, PhD ${ }^{3}$, Doo-Ho Choi, MD, PhD ${ }^{4}$, \\ Min Kyu Kang, MD ${ }^{5}$, Woong-Ki Chung, MD, PhD ${ }^{6}$, Hong-Gyun Wu, MD, PhD ${ }^{1}$ \\ ${ }^{1}$ Department of Radiation Oncology, Seoul National University College of Medicine, Seoul; ${ }^{2}$ Department of Radiation Oncology, \\ Dongnam Institute of Radiological and Medical Sciences, Busan; ${ }^{3}$ Department of Radiation Oncology, Asan Medical Center, \\ Seoul; ${ }^{4}$ Department of Radiation Oncology, Samsung Medical Center, Seoul; ${ }^{5}$ Department of Radiation Oncology, \\ Yeungnam University College of Medicine, Daegu; ${ }^{6}$ Department of Radiation Oncology, \\ Chonnam National University Hwasun Hospital, Huasun, Korea
}

Purpose: This study was designed to evaluate the efficacy and safety of topically applied recombinant human epidermal growth factor (rhEGF) for the prevention of radiation-induced dermatitis in cancer patients.

Materials and Methods: From December 2010 to April 2012, a total of 1,172 cancer patients who received radiotherapy (RT) of more than 50 Gy were prospectively enrolled and treated with EGF-based cream. An acute skin reaction classified according to the Radiation Therapy Oncology Group 6-point rating scale was the primary end point and we also assessed the occurrence of edema, dry skin, or pruritus.

Results: The percentage of radiation dermatitis with maximum grade 0 and grade 1 was 19\% and 58\% at the time of 50 Gy, and it became $29 \%$ and $47 \%$ after completion of planned RT. This increment was observed only in breast cancer patients (from 18\%/62\% to $32 \% / 49 \%$ ). Adverse events related to the EGF-based cream developed in 49 patients (4\%) with mild erythema the most common. Skin toxicity grade $>2$ was observed in 5\% of the patients. Edema, dry skin, and pruritus grade $\geq 3$ developed in $9 \%$, 9\%, and $1 \%$ of the patients, respectively.

Conclusion: Prophylactic use of an EGF-based cream is effective in preventing radiation dermatitis with tolerable toxicity. Further studies comparing EGF cream with other topical agents may be necessary.

Keywords: Radiation induced dermatitis, Cancer, Radiotherapy, Dermatitis, Epidermal growth factor

\section{Introduction}

Radiation-induced skin injury is one of the most common side effects of ionizing radiation and was recognized soon after the discovery of $\mathrm{X}$-rays. Despite the progress in treatment strategies using modern delivery technology and fractionation schedules, this injury still remains a distressing adverse effect of radiotherapy $(\mathrm{RT})$ for treatment of malignancies. The incidence of severe radiation dermatitis even increases after the treatment intensification using concurrent chemotherapy

Received 24 March 2014, Revised 24 June 2014, Accepted 11 August 2014.

Correspondence: Hong-Gyun Wu, MD, PhD, Department of Radiation Oncology, Seoul National University College of Medicine, 103 Daehak-ro, Jongno-gu, Seoul 110-799, Korea. Tel: +82-2-2072-3177, Fax: +82-2-742-2073, E-mail: wuhg@snu. ac.kr

(c) This is an Open Access article distributed under the terms of the Creative Commons Attribution Non-Commercial License (http://creativecommons.org/ licenses/by-nc/3.0/) which permits unrestricted non-commercial use, distribution, and reproduction in any medium, provided the original work is properly cited.

www.e-roj.org 
and accelerated fractionation. Up to $90 \%$ of patients treated with high radiation dose develop dermatitis and some patients experience moist desquamation, which may limit the duration of treatment and the dose delivered and eventually affect the treatment outcome [1-5].

Mild acute radiation dermatitis is usually symptomatically manageable. When erythema and desquamation occurs, topical agents based on natural products can be tried, such as castor oil, balsam of Peru, aloe vera, and calendula, but evidence for their effectiveness is limited. However, clinical management varies widely [6] and there is no standard of care for prevention and control of the progression of radiation dermatitis.

Recently, biologic element-based agents have been investigated for their enhancement of wound healing; these include various growth factors, tacrolimus, and pimecrolimus [2]. Epidermal growth factor (EGF), one of the key elements that function in the wound healing process, has been reported to be protective or therapeutic against mucositis or skin injury induced by radiation in an animal model [7] and in clinical settings $[8,9]$. In Korea, an EGF-based cream (Easydew CR; Daewoong Pharmaceutical, Seoul, Korea) has been used to prevent or alleviate radiation dermatitis. This study investigated the safety and effectiveness of using topical EGF cream for the prevention of radiation dermatitis in patients with cancer.

\section{Materials and Methods}

\section{Patient population}

This prospective, observational study was conducted at 21 centers in Korea between December 2010 and April 2012. Patients aged $\geq 18$ years, affected by histologically confirmed malignancies, and treated with RT ( $\geq 50$ Gy) were eligible for the study. Exclusion criteria were history of connective tissue disorder, such as systemic lupus erythematosus, life expectancy $<3$ months, and uncooperative status, such as dementia or drug abuse. A total of 1,172 patients were recruited initially; 7 did not apply the EGF-based cream, 23 had no assessment at the time of RT of $50 \mathrm{~Gy}$, and 4 dropped out of the study; therefore 1,138 patients were investigated with this protocol (Fig. 1). The studies were approved by the local Institutional Review Board and all patients were provided written informed consent before enrollment. All data were collected and analyzed centrally.

\section{EGF-based cream treatment}

The EGF-based cream contains $0.005 \%$ recombinant human EGF with the wound healing property complemented by the presence of additional ingredients, such as ceramide, hyaluronic acid, Inca omega oil, Portulaca oleracea extract, mango butter, and meadowfoam oil. This cream was applied to the radiation portal skin twice a day, from day 1 to the last day of RT. Patients were advised to wash the cream off prior to RT. No other prophylactic creams or lotions were allowed and further applications were ceased if an adverse reaction occurred due to the EGF-based cream. Compliance in applying the cream was evaluated weekly by the treating radiation oncologist.

\section{Assessment}

The treating radiation oncologists examined the patients at weekly intervals from 1 week before RT to after RT. Skin toxicity was scored using the modified Radiation Therapy Oncology Group (RTOG) criteria-grade 0, no reaction; grade 1, erythema; grade 2 , dry desquamation; grade 3 , focal wet desquamation; grade 4, confluent wet desquamation; and grade 5 , necrosis of the skin. This scale of radiation dermatitis was modified with a representative sign to reduce interobserver variations. The primary endpoint was the maximum grade of radiation dermatitis. The effectiveness of the EGF-based cream was

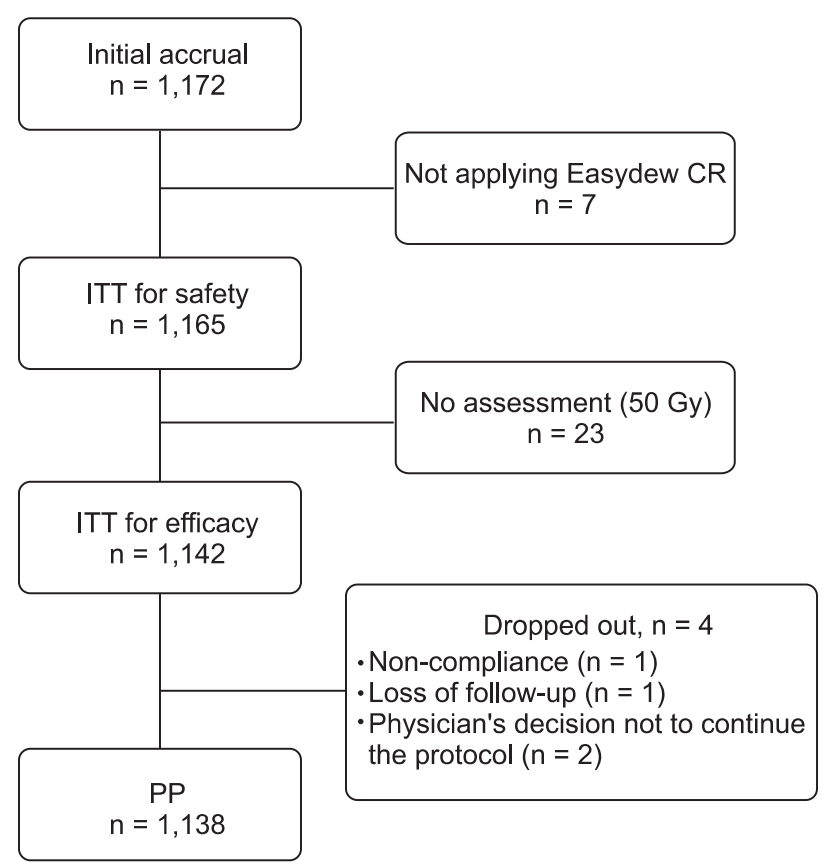

Fig. 1. Patient disposition. IT, intention-to-treat analysis; PP, perprotocol analysis. 
Table 1. Patient characteristics

\begin{tabular}{lc}
\hline \multicolumn{1}{c}{ Characteristic } & Value \\
\hline Age $(y r)$ & $52.1 \pm 11.4(18-90)$ \\
Sex & $201(17.6)$ \\
Male & $941(82.4)$ \\
Female & \\
Site of cancer & $834(73.0)$ \\
Breast & $148(13.0)$ \\
Head and neck & $160(14.0)$ \\
Others ${ }^{\text {a) }}$ & \\
Mode of RT delivery & $591(51.8)$ \\
Photon & $53(4.6)$ \\
Electron & $498(43.6)$ \\
Photon with electron & $58.6 \pm 5.59(50-75)$ \\
Dose of RT (Gy)
\end{tabular}

Values are presented as mean \pm standard deviation (range) or number (\%).

$\mathrm{RT}$, radiotherapy.

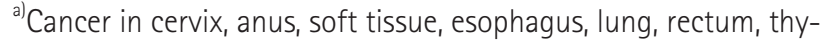
mus.

assessed by comparing the baseline skin condition with the condition at the time of receiving 50 Gy of $\mathrm{RT}$ and again at the end of the planned RT. The mean maximum grade scores according to radiation total dose were also compared to assess the prophylactic effect of the EGF-based cream for patients receiving high doses of RT. Other toxicities were assessed for the development of edema, dry skin and pruritus. The status of pruritus was scored using the following criteria-grade 0 , no pruritus; grade 1, mild or localized, relieved spontaneously; grade 2, mild or localized, relieved by local measures; grade 3 , intense or widespread, relieved by systemic measures; and grade 4, intense or widespread and poorly controlled despite treatment. The adverse effects from the EGF-based cream were assessed to identify the severity and relevance to the study material. Due to the lack of randomization and the different therapy schemes, only descriptive statistics are provided.

\section{Results}

\section{Patient and treatment characteristics}

In the total patient population, 1,142 patients completed the planned RT with more than 50 Gy and were evaluated for the grade of radiation dermatitis according to their followup schedule. Four patients were dropped from the studyfor non-compliance $(n=1)$, loss of follow-up $(n=1)$, and the physician's decision not to continue the protocol $(n=2)$.
Because we did not exclude these patients from the efficacy analysis, all patients were evaluable.

Table 1 presents relevant patient characteristics. The percentage of female patients was $82.4 \%$ and the median age of the study population was 51 years. The radiation dose ranged from 50-75 Gy, and the median dose value was $60 \mathrm{~Gy}$. The most common sites of RT were breast, head and neck, and pelvis. Radiotherapy was delivered using a photon beam in 52\% of patients, an electron beam in 5\%, and combined photon and electron beams in $43 \%$.

\section{Radiation dermatitis grade}

Radiation dermatitis developed in 925 patients (79.9\%) during and after RT. At the time of $50 \mathrm{~Gy}$ of $\mathrm{RT}$, radiation dermatitis of maximum grades 1, 2, 3, and 4 developed in 58.3\%, 17.6\%, $4.7 \%$, and $0.3 \%$ of the patients, respectively. In comparison, after the planned $\mathrm{RT}$, radiation dermatitis of the maximum grades $1,2,3$, and 4 were observed in 46.6\%, 18.0\%, 5.5\%, and $0.9 \%$ of the patients, respectively (Table 2). The proportion of the patients assessed as normal increased from 19.1\% (at the time of $50 \mathrm{~Gy}$ ) to $29 \%$ (after RT) in the entire cohort. However, the grade 3-4 dermatitis developed more frequently after RT among the patients who were treated for non-breast disease. We also evaluated skin toxicity according to the total radiation dose dividing the patients into 4 different groups. The mean maximum grade in patients receiving $<55,55-59.9,60-64.9$, and $>65$ Gy was 0.93 (standard deviation, \pm 0.86$), 1.35( \pm 0.7)$, $1.06( \pm 0.76)$, and $1.39( \pm 1.02)$ (Fig. 2). The incidence of edema and dry skin was $9.4 \%$ and $9.3 \%$, and pruritus grade $\geq 3$ was observed in $1.1 \%$ patients (Table 3 ).

\section{Safety}

The EGF-based cream was well tolerated. Potential adverse effects of the cream were observed in 55 of 1,165 patients $(4.72 \%)$ who applied the cream at least 1 or more timeserythema in 31 patients, pruritus in 12 patients, irritation in 5 patients, engorgement in 4 patients, confluent dermatitis in 2 patients, and heat sensation in 1 patient. Only 12 patients experienced intermediate-grade events, and no patient dropped out of the study due to adverse events. The relevance between adverse events and the EGF-based cream was retrospectively analyzed, and 9 events were considered as 'relevant' or 'possibly relevant'. The safety of the EGFbased cream during the entire treatment period was assessed on the last visit by the patients; $99.2 \%$ of the patients were categorized as 'safe' and $0.8 \%$ as 'suspicious or unsafe' at the 
Table 2. Maximum radiation dermatitis in detail through the study

\begin{tabular}{|c|c|c|c|c|c|}
\hline & \multicolumn{5}{|c|}{ Radiation dermatitis } \\
\hline & Grade 0 & Grade 1 & Grade 2 & Grade 3 & Grade 4 \\
\hline \multicolumn{6}{|l|}{ All $(n=1,142)$} \\
\hline Week 0 & 1,132 (99.1) & $9(0.8)$ & $1(0.1)$ & $0(0)$ & $0(0)$ \\
\hline Week 5 (50 Gy) & 217 (19.1) & $663(58.3)$ & $200(17.6)$ & $55(4.7)$ & $3(0.3)$ \\
\hline Week 8 (end) & $331(29.0)$ & $532(46.6)$ & 206 (18.0) & $63(5.5)$ & $10(0.9)$ \\
\hline \multicolumn{6}{|l|}{ Breast $(n=838)$} \\
\hline Week 0 & 832 (99.3) & $5(0.6)$ & $1(0.1)$ & $0(0)$ & $0(0)$ \\
\hline Week 5 & $147(17.5)$ & $523(62.4)$ & $146(17.4)$ & $22(2.6)$ & $0(0)$ \\
\hline Week 8 & $268(32.0)$ & $410(48.9)$ & $139(16.6)$ & $21(2.5)$ & $0(0)$ \\
\hline \multicolumn{6}{|c|}{ Non-breast $(n=304)$} \\
\hline Week 0 & $300(98.7)$ & $4(1.3)$ & $0(0)$ & $0(0)$ & $0(0)$ \\
\hline Week 5 & $71(23.4)$ & $140(46.1)$ & $55(18.1)$ & $35(11.5)$ & $3(1.0)$ \\
\hline Week 8 & $63(20.7)$ & $122(40.1)$ & $67(22.0)$ & 42 (13.8) & $10(3.3)$ \\
\hline
\end{tabular}

Values are presented as number (\%).

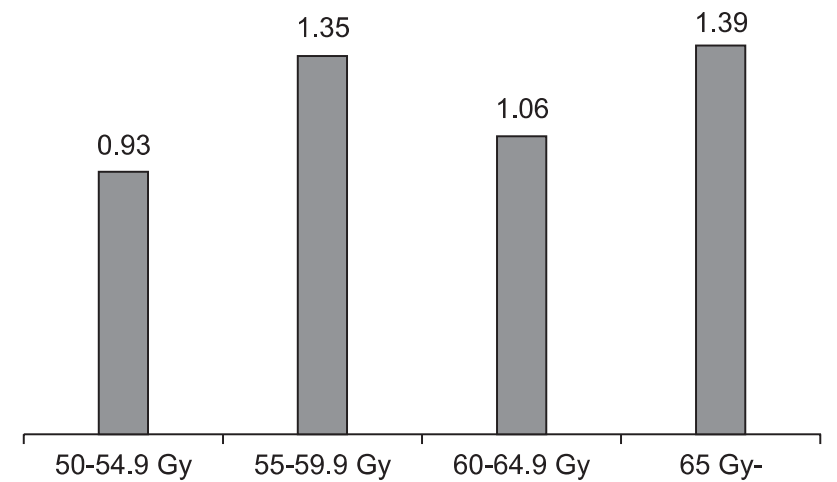

Fig. 2. Mean value of radiation dermatitis grade according to radiation dose range.

discretion of the treating physician.

\section{Discussion and Conclusion}

Radiation dermatitis remains the most common acute complication resulting from RT. The process of radiationinduced skin damage is mediated by free radicals that cause DNA damage, which is followed by inflammatory cell recruitment and tissue injury [2]. The response of normal tissue to ionizing radiation includes inflammation, epithelial regeneration, granulation tissue formation, matrix deposition, and remodeling [10]. Although the pathophysiology and underlying biologic mechanism are not yet fully understood, growth factors are considered to promote wound healing by stimulating cell proliferation [11]. EGF is one of the key growth factors and stimulates the proliferation of human
Table 3. Radiation toxicity other than dermatitis

\begin{tabular}{ccc}
\hline Toxicity & No. $(\%)$ & $95 \% \mathrm{Cl}^{2)}$ \\
\hline Edema & & {$[7.74,11.21]$} \\
Yes & $107(9.37)$ & \\
No & $1,035(90.63)$ & \\
Dry skin & & {$[7.76,11.12]$} \\
$\quad$ Yes & $106(9.28)$ & \\
No & $1,036(90.72)$ & \\
Pruritus & & {$[98.06,99.39]$} \\
$\quad$ Grade 2 & $1,129(98.86)$ & \\
$\geq$ Grade 3 & $13(1.14)$ & \\
\hline
\end{tabular}

${ }^{a}$ Exact confidence interval.

fibroblasts and keratinocytes during the healing process $[11,12]$. In practice, the use of topical EGF has been proven effective in treating diabetic foot ulcers [13] and the effectiveness of EGF in the healing process after radiation injury has also been reported in pre-clinical or clinical models [7-9].

The evidence at present is insufficient to arrive at consensus guidelines for the use of EGF in clinical practice for radiation dermatitis management $[14,15]$. A few randomized phase III trials have reported a superior preventive effect of topical agents, such as corticosteroid [16], calendula [17], and silverbased dressings [18] on radiation dermatitis development. However, the use of topical steroid is controversial because this can cause thinning of the skin and introduce bacterial infection. Calendula ointment and silver-based dressings are not easily applicable because of their solid property and the need for daily dressing tasks. No study has validated the use 
of these candidate materials for their efficacy and safety with a large study population or in a prospective manner. In Korea, a recombinant human EGF-based cream had been developed and used to prevent or minimize radiation dermatitis in cancer patients [19]. Despite the enthusiasm for EGF application, no large-scale prospective trial has been conducted on the use of EGF-based medication in preventing or minimizing radiation dermatitis. The present study represents the first prospective study in a cancer patient cohort of 1,172 men and women.

The incidence and the severity of radiation dermatitis in our study were significantly different than the results reported in other studies. In our study, the incidence of the radiation dermatitis after RT was 46.6\%, 18.0\%, 5.5\%, and 0.9\% from grade 1 to grade 4 , respectively. The maximum grade $\geq 2$ toxicity developed less frequently when compared with that of the control group in two large randomized trials $[17,20]$, where its incidence ranged from 60\% to 85\%. Different study designs, including evaluation scales, may have attributed to the discrepancy observed in radiation toxicity rates, and the lower rate of radiation dermatitis in our study could be related to variable factors relating radiation dermatitis, such as RT dose, RT schedule, use of chemotherapy, smoking, underlying disease, and heterogeneous patient population. However, the proportion of patients assessed as normal in our study increased by $10 \%$ from the time of 50 Gy to the end of RT, in contrast to these other studies. This increase in the proportion should be interpreted with a caution; it was demonstrated only in breast cancer patients and most of them had been irradiated with reduced RT field after 50 Gy of whole breast RT that might be closed associated with a substantial reduction of radiation damage. Nonetheless, patients suffering grade 3 dermatitis did not increase in breast cancer group and it may reflect a protective or healing effect of EGF on the skin near the tumor bed that was irradiated with high dose RT until end of the treatment. Similarly, the fast recovery from radiation dermatitis in the study patients supports the positive effect of EGF. In addition, the development of radiation dermatitis was independent to total radiation dose, which may have been an outcome of the preventive effect of EGF regarding progression of the damaged skin in response to further irradiation.

The treatment was well tolerated, and no severe adverse effects or mortality were observed in the study population. However, one major concern is the potential cancer-enhancing properties of EGF used for preventive purposes. Recent reports have indicated that exogenously administered EGF does not stimulate cancer cell proliferation in tumor-bearing xenograft mice [21], but other reports have shown opposite results [2224]. The total amount of EGF in the EGF-based cream is far lower than the $15 \mu \mathrm{g}$ EGF concentration used in previous studies [22]; therefore, we suppose that local application of Easydew CR is safe. In addition, we found no evidence in a recent literature review to indicate that EGF initiated tumorigenesis [25] and the review infers that carcinogenesis is not a significant concern with locally applied EGF.

Our study has some limitations arising mainly from the study design. First, the skin toxicities were assessed by the treating physician, which means that the incidence or severity of radiation dermatitis might be underestimated or overestimated. However, the RTOG is the most popular scoring system for radiation dermatitis, and we modified the evaluation criteria of the study to a 6-point scale to allow the observer to distinguish the grade of radiation toxicity more distinctly. Second, radiation dermatitis was assessed by limited objective tools and it would have been valuable to also evaluate variables such as inflammatory responses or the barrier function of the skin [26]. Third, we had a total observation period of 8 weeks after the start date of RT, so it was not possible to record the late development as well as the healing process of radiation dermatitis after EGF treatment. Finally, the RT doses to the skin were not surveyed in a practical method but only supposed from the described RT doses.

In conclusion, this multicenter prospective cohort study of 1,172 patients undergoing RT for variable malignancies showed that a recombinant human EGF-based cream could be safely applied to prevent or alleviate radiation dermatitis. The study lacked a design to compare the effects with conservative management or with other topical agents, so future trials are warranted with a placebo-controlled randomized design to confirm the effectiveness of EGF-based cream as a treatment for radiation dermatitis.

\section{Conflict of Interest}

No potential conflict of interest relevant to this article was reported.

\section{Acknowledgments}

We thank the following institutions from which this work originated: Pusan National University Hospital, Soonchunhyang University Hospital, Wonkwang University School of Medicine 
Et Hospital, Dong-A University Hospital, Inje University Ilsan Paik Hospital, Inje University Busan Paik Hospital, Inje University Sanggye Paik Hospital, Hallym University Medical Center, Chung-Ang University Hospital, Keimyung University Dongsan Medical Center, Eulji University Hospital, Chungnam National University Hospital, The Catholic University of Korea Seoul St. Mary's Hospital, The Catholic University of Korea Incheon St. Mary's Hospital, Dankook University Hospital, Inha University Hospital for data collection.

\section{References}

1. Harper $J \mathrm{~L}$, Franklin $\mathrm{LE}_{\mathrm{E}}$ Jenrette $J \mathrm{M}$, Aguero EG. Skin toxicity during breast irradiation: pathophysiology and management. South Med J 2004;97:989-93.

2. Hymes SR, Strom EA, Fife C. Radiation dermatitis: clinical presentation, pathophysiology, and treatment 2006. J Am Acad Dermatol 2006;54:28-46.

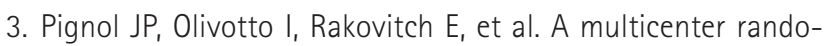
mized trial of breast intensity-modulated radiation therapy to reduce acute radiation dermatitis. J Clin Oncol 2008;26:208592.

4. Fisher J, Scott C, Stevens R, et al. Randomized phase III study comparing Best Supportive Care to Biafine as a prophylactic agent for radiation-induced skin toxicity for women undergoing breast irradiation: Radiation Therapy Oncology Group (RTOG) 97-13. Int J Radiat Oncol Biol Phys 2000;48:1307-10.

5. Lee SY, Kwon HC, Kim JS, Lee HK. An analysis of the incidence and related factors for radiation dermatitis in breast cancer patients who received radiation therapy. J Korean Soc Ther Radiol Oncol 2010;28:16-22.

6. Bolderston A, Lloyd NS, Wong RK, Holden L, Robb-Blenderman L; Supportive Care Guidelines Group of Cancer Care Ontario Program in Evidence-Based Care. The prevention and management of acute skin reactions related to radiation therapy: a systematic review and practice guideline. Support Care Cancer 2006;14:802-17.

7. Lee SW, Jung KI, Kim YW, Jung HD, Kim HS, Hong JP. Effect of epidermal growth factor against radiotherapy-induced oral mucositis in rats. Int J Radiat Oncol Biol Phys 2007;67:1172-8.

8. Ryu SH, Kim YH, Lee SW, Hong JP. The preventive effect of recombinant human growth factor (rhEGF) on the recurrence of radiodermatitis. J Radiat Res 2010;51:511-7.

9. Wu HG, Song SY, Kim YS, et al. Therapeutic effect of recombinant human epidermal growth factor (RhEGF) on mucositis in patients undergoing radiotherapy, with or without chemotherapy, for head and neck cancer: a double-blind placebocontrolled prospective phase 2 multi-institutional clinical trial.
Cancer 2009;115:3699-708.

10. Denham JW, Hauer-Jensen M. The radiotherapeutic injury: a complex 'wound'. Radiother Oncol 2002;63:129-45.

11. Olascoaga A, Vilar-Compte D, Poitevin-Chacon A, ContrerasRuiz J. Wound healing in radiated skin: pathophysiology and treatment options. Int Wound J 2008;5:246-57.

12. Haubner F, Ohmann E, Pohl F, Strutz J, Gassner HG. Wound healing after radiation therapy: review of the literature. Radiat Oncol 2012;7:162.

13. Hong JP, Jung HD, Kim YW. Recombinant human epidermal growth factor (EGF) to enhance healing for diabetic foot ulcers. Ann Plast Surg 2006;56:394-400.

14. Chan RJ, Larsen E, Chan P. Re-examining the evidence in radiation dermatitis management literature: an overview and a critical appraisal of systematic reviews. Int J Radiat Oncol Biol Phys 2012;84:e357-62.

15. Zhang $Y$, Zhang $S$, Shao $X$. Topical agent therapy for prevention and treatment of radiodermatitis: a meta-analysis. Support Care Cancer 2013;21:1025-31.

16. Schmuth $M$, Wimmer MA, Hofer $S$, et al. Topical corticosteroid therapy for acute radiation dermatitis: a prospective, randomized, double-blind study. Br J Dermatol 2002;146:98391.

17. Pommier P, Gomez F, Sunyach MP, D'Hombres A, Carrie C, Montbarbon X. Phase III randomized trial of Calendula officinalis compared with trolamine for the prevention of acute dermatitis during irradiation for breast cancer. J Clin Oncol 2004;22:1447-53.

18. Niazi TM, Vuong $T$, Azoulay $L$, et al. Silver clear nylon dressing is effective in preventing radiation-induced dermatitis in patients with lower gastrointestinal cancer: results from a phase III study. Int J Radiat Oncol Biol Phys 2012;84:e305-10.

19. Kong M, Hong SE. Topical use of recombinant human epidermal growth factor (EGF)-based cream to prevent radiation dermatitis in breast cancer patients: a singleblind randomized preliminary study. Asian Pac J Cancer Prev 2013;14:4859-64.

20. Elliott EA, Wright JR, Swann RS, et al. Phase III Trial of an emulsion containing trolamine for the prevention of radiation dermatitis in patients with advanced squamous cell carcinoma of the head and neck: results of Radiation Therapy Oncology Group Trial 99-13. J Clin Oncol 2006;24:2092-7.

21. Choi J, Moon SY, Hong JP, Song JY, Oh KT, Lee SW. Epidermal growth factor induces cell death in the absence of overexpressed epidermal growth factor receptor and ErbB2 in various human cancer cell lines. Cancer Invest 2010;28:50514.

22. Geissler $C_{1}$ Hambek $M$, Eckardt $A$, et al. The role of recombinant epidermal growth factor and serotonin in the stimulation of tumor growth in a SCCHN xenograft model. Oncol Rep 
2012;28:785-90.

23. Ozawa S, Ueda M, Ando N, Abe O, Hirai M, Shimizu N. Stimulation by EGF of the growth of EGF receptor-hyperproducing tumor cells in athymic mice. Int J Cancer 1987;40:706-10.

24. Ginsburg E, Vonderhaar BK. Epidermal growth factor stimulates the growth of A431 tumors in athymic mice. Cancer Lett 1985;28:143-50.
25. Berlanga-Acosta J, Gavilondo-Cowley J, Lopez-Saura P, et al. Epidermal growth factor in clinical practice: a review of its biological actions, clinical indications and safety implications. Int Wound J 2009;6:331-46.

26. Schmuth $M$, Sztankay $A$, Weinlich $G$, et al. Permeability barrier function of skin exposed to ionizing radiation. Arch Dermatol 2001;137:1019-23. 